\title{
THE DILATATION OF THE UPPER URINARY TRACT IN PARAPLEGICS
}

\author{
By Professor R. Ascoli and Professor L. Franch \\ Centro Traumatologico di Rieducazione, Milano
}

I THOUGHT it would be useful to make a statistical survey of the incidence of the dilatations of the upper urinary tract in paraplegics after traumatism of the spinal cord.

It is a well-known fact that the most severe complications of cystoplegia after traumatism of the cord are (I) the often very dangerous infections of the upper. urinary tract, and (2) the dilatations of the upper urinary tract.

Up till a few years ago such dilatations were considered in the classic works on the subject as very frequent, although not many statistical data existed, but authors gave particular emphasis to their extremely dangerous nature, inasmuch as they thought these dilatations could constitute a pre-stage to renal failure.

It should not be forgotten that as recently as a few years ago, the most frequent causes of death in paraplegics were urological complications (70 to 90 per cent.).

Our statistical survey was carried out on the clinical material of the Traumatologic Centre of INAIL in Milan (the Italian National On-job Accident Insurance Institute); we reviewed a considerable number of urographies in paraplegics during the period from 1957 to 1966.

It seemed to us that a survey would be of considerable interest, inasmuch as it represented the contribution of a given paraplegic centre, where the urologic treatment of the paraplegic presents peculiar characteristics, and where a particular type of treatment has been standardised.

It is a well-known fact that not all cord specialists use the same methods of treatment and reablement. We all know that in some centres the system of catheterisation is preferred, while elsewhere the indwelling catheter or the methods of the temporarily obstructed catheter or the method of tidal drainage are systematically used.

In our view, therefore, the concept of comparing the different degrees of dilatation of the upper urinary tract could be a sign of the greater or lesser efficacy of the kind of therapy used. In other words, we think it would be very interesting if our statistical contribution, which originates from a service where particular therapeutic concepts are applied, were to be followed up by a statistical contribution originating from other services where different therapeutic concepts are applied.

We would recall that in a paper of his in 1954, Comarr stated that when examining the urographies of 3is medullaries, he encountered dilatation of the upper urinary tract in 26 per cent. of cases.

More recently Damanski gave the results of a survey he carried out on I62 paraplegic patients: he found dilatations in the upper urinary tract in 43 per cent. of cases.

Urological pathogenetic factor number one, however, is in our opinion clearly the persistent increase in endovesical pressure resulting from retention, an increase which in the absence of adequate therapy is more or less constant in these patients mainly during the first few months after the trauma. 
The importance of this factor is limited when right from the start the patient has been given suitable urological treatment (periodical catheterisation or indwelling catheters, or tidal drainage), but it is very great when such therapy has not been applied in the proper way and at the proper time.

Factor number two is the impairment of the nervous pathways that regulate the function of the urethro-vesical valve.

A pathogenetic factor of minor importance is the presence of acute or chronic cystitis which, inducing an irritative condition of the bladder with resulting hypertonia, may be the cause of sharp contractions and sharp and sudden rises in endovesical pressure.

The same may be said for the presence of any stones in the bladder.

The principles on which we have worked in studying our material were as follows: we distinguished three degrees of dilatation of the endorenal cavity.

By first degree dilatations we mean conditions of simple pyelectasis, barely more severe than conditions we might describe as physiological.

By second degree dilatation we mean a condition where there is an obvious ectasis of the pelvis and of the calyces, with a tendency for the latter to assume an $a$ boule shape.

By third degree dilatations we mean cases of true hydronephrosis.

We have taken the following factors into consideration: (I) age, (2) level of the lesion, (3) time elapsed between the date of the trauma and the date of the examination, (4) possible concomitance of reflux. We studied 173 cases. We found dilatations in 35 cases $(20 \cdot 2$ per cent). Of these, 27 cases belonged to the first group, namely those with slight dilatation ( 57 per cent. of the total number of cases of dilatation). Fourteen cases belonged to the second group of dilatations of average seriousness (equivalent to 40 per cent. of the total number of dilated cases).

Only one case ( $2 \cdot 8$ per cent.) belonged to the third group (true hydronephrosis).

As regards age, we divided our patients into three groups. The first group included patients from 0 to 20 years old, the second from 21 to 40 and the third over 40.

With regard to the level of the lesion, we distinguished three groups, according to whether the cervical, dorsal or lumbar column was involved.

With regard to the relation between time elapsing since the trauma and frequency of the dilatations, we divided our material into four groups.

The first group included the cases of dilatation found during the first six months from the date of the trauma.

The second group included the cases of dilatation found from six months to two years after the trauma.

The third group included cases of dilatation found from two to four years after the trauma.

The last group included cases of dilatation found at more than four years from the date of the trauma.

If we wish to compare the above data, or the respective percentages in relation to the various factors examined, we may state:

(I) With regard to age, we feel it may be concluded that there is a slightly greater tendency towards dilatation of the upper urinary tracts in older as compared with young persons.

(2) With regard to the height of the lesion, from our study it did not emerge 
that this factor had an appreciable influence on the frequency of dilatations in the upper urinary tracts.

(3) With regard, however, to the time elapsing from the trauma, it would seem from our survey that the percentage of dilated cases is much higher in the fourth group including patients observed at over four years from the date of the trauma, as compared with those observed after a relatively short time.

The clinical importance of the research we have done seems to lie in the discovery that the overall percentage of dilatations we encountered (corresponding to 20 per cent.) is considerably lower than that recorded by other well-known authors (Comarr 26 per cent., Damanski 43 per cent.); above all if we consider that in over half the cases the dilatation was slight.

We have already said that it is fairly clear that there must be some connection between the difference in frequency and the therapeutic methods used.

At our Traumatologic Centre in Milan a method is used that involves systematic treatment by tidal drainage during the months immediately following the trauma: we know that this system is, however, not adopted today by the majority of authors.

We are inclined to think that the relatively low percentage of dilatations we found in our material is due to the therapeutic method we used-a method we have described in detail on many occasions.

\section{SUMMARY}

Analysis of a great number of urographies of paraplegics was made in order to establish the frequency of dilatations of upper urinary tract. Age, level of the lesions, time elapsed from the date of the trauma, were taken into consideration. Dilatations were found in 35 cases ( 20.2 per cent.) but 27 of these cases ( 57 per cent.) belonged to the group of the very slight dilatations, I 4 cases ( 40 per cent.) belonged to the group of average seriousness. Only one case belonged to the group of the true hydronephrosis. 